\title{
The H-beta emission line profile of Arakelian 120
}

\section{Citation}

Foltz, C. B., B. J. Wilkes, and B. M. Peterson. 1983. "The H-Beta Emission Line Profile of Arakelian 120." The Astronomical Journal 88 (December): 1702. doi:10.1086/113462.

\section{Published Version}

10.1086/113462

\section{Permanent link}

http://nrs.harvard.edu/urn-3:HUL.InstRepos:32955258

\section{Terms of Use}

This article was downloaded from Harvard University's DASH repository, and is made available under the terms and conditions applicable to Other Posted Material, as set forth at http:// nrs.harvard.edu/urn-3:HUL.InstRepos:dash.current.terms-of-use\#LAA

\section{Share Your Story}

The Harvard community has made this article openly available.

Please share how this access benefits you. Submit a story.

Accessibility 


\title{
THE H $\beta$ EMISSION LINE PROFILE OF ARAKELIAN 120
}

\author{
Craig B. Foltz ${ }^{\text {a) }}$ and Belinda J. Wilkes \\ Steward Observatory, University of Arizona, Tucson, Arizona 85721 \\ Bradley M. Peterson ${ }^{\text {b) }}$ \\ Perkins Observatory, The Ohio State and Ohio Wesleyan Universities, Delaware, Ohio 43015 \\ Received 29 March 1983; revised 30 June 1983
}

\begin{abstract}
A high-quality $(30<\mathrm{S} / \mathrm{N}<100)$, high-resolution $(\sim 1.8 \AA)$ spectrum of the photometrically and spectroscopically variable Seyfert 1 galaxy Arakelian 120 has been obtained in the wavelength region including $\mathrm{H} \beta$. The observed $\mathrm{H} \beta$ line profile can be considered as a benchmark for future line profile studies of this interesting object. The origin of the extended red wing of $\mathrm{H} \beta$ is discussed.
\end{abstract}

\section{INTRODUCTION}

The recent theoretical studies of Capriotti, Foltz, and Peterson (1982) and Blandford and McKee (1982) emphasize the importance of the study of the temporal behavior of the broadline profiles in Seyfert 1 galaxies as a diagnostic of the structure and dynamics of the broadline emitting gas. Arakelian 120 is an excellent candidate for such a study. Long known to be both spectroscopically and photometrically variable, Akn 120 is bright enough to be accessible to telescopes of modest aperture. It has been shown that the $\mathrm{H} \beta$ emission line responds rapidly to changes in the luminosity of the continuum source (Peterson et al. 1983, and references cited therein).

Most of the previous work on the nature of the spectroscopic variations of Akn 120 have been based on the variations of the equivalent widths of $\mathrm{H} \beta$ and [O III] $\lambda \lambda 4959,5007$, and though $\mathrm{H} \beta$ profile variations have been clearly observed, the signal-to-noise ratios $(\mathrm{S} / \mathrm{N})$ and resolution of these observations are, in general, not superior.

In order to test the suggestions of Capriotti, Foltz, and Peterson (1982) that variations in the continuum source will be manifest as structure in the emission line profiles, it is essential that any irregularities in the profiles are readily identifiable and that the temporal behavior of these individual small-scale features be closely monitored. In this contribution a high $\mathrm{S} / \mathrm{N}$, high-resolution profile of $\mathrm{H} \beta$ in Akn 120 is presented. Our intent is to provide a benchmark line profile against which future profiles can be compared.

\section{OBSERVATIONS}

Akn 120 was observed for a total of $14400 \mathrm{~s}$ on the night of 1983 January 9 (U.T.) with the Boller and Chi-

a) Current address: Department of Astronomy, University of Illinois, Urbana, Illinois 61801.

${ }^{b)}$ Mailing address: Department of Astronomy, Ohio State University, 174 West 18th Avenue, Columbus, Ohio 43210.

1702 Astron. J. 88 (12), December 1983

0004-6256/83/121702-05\$00.90 vens spectrograph equipped with a dual beam photoncounting Reticon detector mounted on the Steward Observatory $2.3-\mathrm{m}$ telescope. The spectrograph entrance apertures were circular holes with a projected diameter of 2.5 arcsec. All observations were made during conditions of good transparency and fair seeing.

Though the data were reduced following fairly standard procedures, the desired very high $\mathrm{S} / \mathrm{N}$ of the spectrum dictates some additional discussion.

Wavelength calibration. A fourth-order polynomial was fitted to the positions of $35 \mathrm{He}-\mathrm{Ar}$ comparison lines with a standard deviation of $0.12 \AA$. Observations of comparison spectra were liberally interspersed with the observations of Akn 120. These were carefully checked for systematic shifts due to physical and/or magnetic flexure. Since no shifts greater than $0.1 \AA$ were found; the data were coadded on a pixel-to-pixel basis.

Removal of pixel-to-pixel sensitivity variations. Coadded spectra were divided by spectra of a quartz incandescent lamp in order to remove pixel-to-pixel sensitivity variations which have an amplitude of $2 \%-3 \%$ in the raw data. The lamp spectra were broken into several short integrations enabling quotients of independent lamp observations to be formed. Their deviations from unity were consistent with photon-counting statistics to within $\sim 1 \%$.

Extinction corrections and flux calibration. The effects of atmospheric extinction were removed using a mean extinction curve appropriate for Kitt Peak. Reduction of the data to relative flux units (energy $/ \mathrm{cm}^{2} / \mathrm{s}$ / $\mathrm{Hz}$ ) was facilitated by referencing the data to observations of the white dwarf EG20 whose spectrometric properties are known (Oke 1974). Flux calibration points fully covered the observed spectral range, running from 4420 to $5620 \AA$ at $40-\AA$ intervals. Due to the combination of fair seeing and small entrance apertures, no attempt was made to determine the absolute flux scale of the final spectrum.

Since the Reticon scanner is a dual-beam instrument, data from each aperture were reduced separately, binned into a common wavelength scale, averaged and (c) 1983 Am. Astron. Soc. 1702 


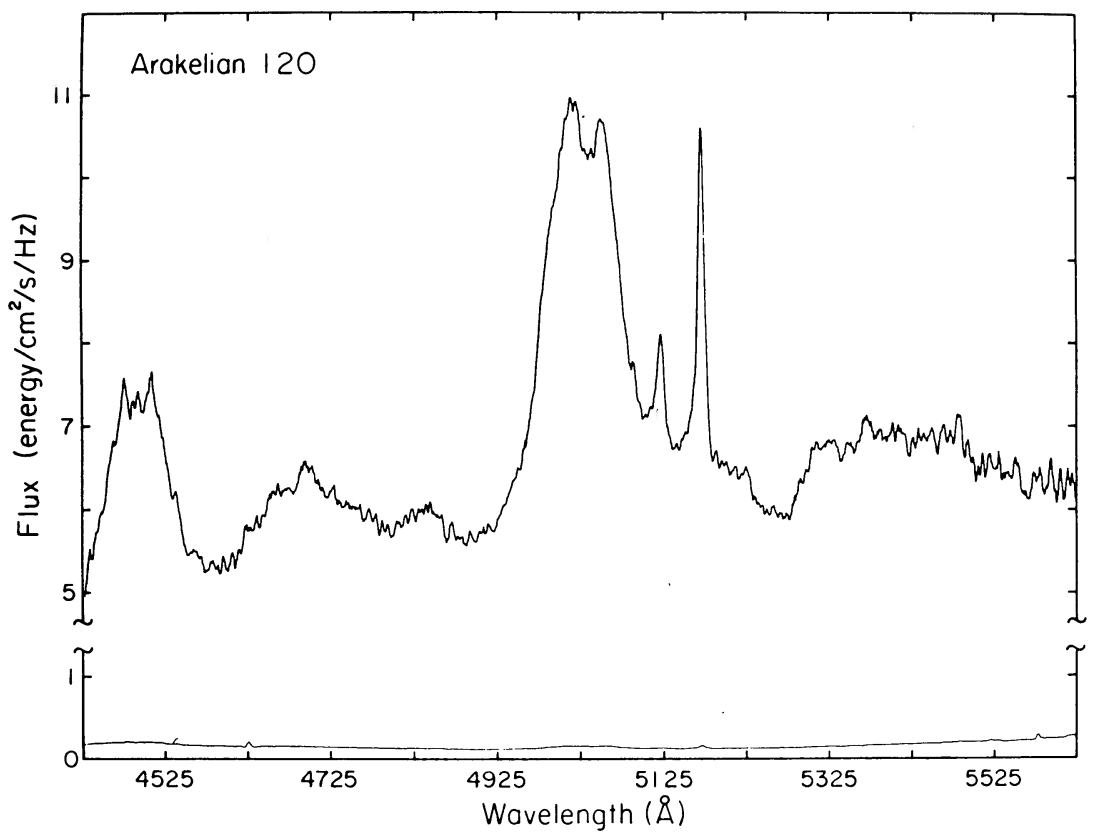

FIG. 1. Spectrum of Arakelian 120 obtained with the Steward Observatory B \& C spectrograph with photon-counting Reticon detector. The lower curve shows the $2 \sigma$ noise level plotted on the same scale.

smoothed via convolution with a 3-pixel-wide boxcar. The resolution of the raw data as determined by the FWHM of an unresolved comparison line was $1.5 \AA$. After rebinning and smoothing, this was degraded to $\sim 1.8 \AA$ as determined by performing the same operations on the comparison lamp spectrum as were performed in the reduction of Akn 120.

The analysis of the quartz lamp spectra indicates that the data are photon noise limited. Assuming that this was the only source of noise, the variance of the data was propagated through the reduction procedure. Sources of noise considered were photon noise in observations of the object, sky, and quartz lamp.

In Fig. 1 our spectrum of Akn 120 is presented along with the spectrum of the $2 \sigma$ level of the noise. The actual value of $\mathrm{S} / \mathrm{N}$ varies from $>30$ at the ends of the spectrum to $>100$ at the peak of the $\mathrm{H} \beta$ profile.

\section{DISCUSSION}

The spectrum in Fig. 1 was examined for weak emission features. It is found that, in general, most of the irregularities in the spectrum can be identified with individual transitions or blends of transitions mostly due to permitted multiplets of Fe II. Also identified are [Ca v] $\lambda$ 5308, [N I] $\lambda$ 5199, [Fe VI] $\lambda$ 5177, and possibly [A IV] $\lambda \lambda 4711,4740$. The only identifiable irregularity within the $\mathrm{H} \beta$ profiles is $\mathrm{Fe}$ II $\lambda 4924$. Due to the severe intrinsic blending of the weak features, no attempt was made to measure their line strengths.

The $\mathrm{H} \beta$ equivalent width was measured in the manner described by Peterson et al. (1983) to be $110 \pm 5 \AA$ in the rest frame of the [O III] lines $(z=0.03248)$. This measurement includes the extended red wing of $\mathrm{H} \beta$ after the [O III] lines have been excised via interpolation of the red wing of $\mathrm{H} \beta$. The relative strength of [O III] $\lambda 5007$ was measured to be $0.083 \pm 0.002(\mathrm{H} \beta=1.000)$. Both $\lambda 5007$ and $\lambda 4959$ were measured to arrive at this value, assuming $F(\lambda$ 5007): $F(\lambda$ 4959)::3:1.

Comparison of these line measurements with those of Peterson et al. (1983) reveals that the optical continuum level has increased significantly (by $\sim 0.5 \mathrm{mag}$ which is greater than $\sim 3 \sigma$ ) since the period between 1982 October-December. The $\mathbf{H} \beta$ flux, however, appears to be comparable to that observed late in 1982, which implies that the effect of the recent outburst has not yet been communicated to the entire broad-line emitting region.

The $\mathrm{H} \beta$ profile itself is remarkably free of small scale irregularities. A strong bump is observed at $\sim 4890$ (rest), approximately $1800 \mathrm{~km} / \mathrm{s}$ redward of line center as defined in the rest frame of the [O III] lines. It is of interest to note that a feature was reported at this displacement by Foltz et al. (1981). A similar feature is seen in the spectrum observed by Osterbrock and Phillips (1977) in 1976 November and in most of the $\mathrm{H} \beta$ profiles presented by Capriotti, Foltz, and Peterson (1982). Foltz et al. (1981) point out a second feature at $\sim-1300 \mathrm{~km} / \mathrm{s}$. Though this feature is not resolved as a separate peak in our profile, the additional inflection in the blue wing giving rise to a shoulder on the line suggests its presence at roughly the same wavelength.

We now turn our attention to the extended red wing of $\mathrm{H} \beta$ which is clearly apparent in Fig. 1 beneath the [O III] $\lambda \lambda 4959,5007$ profiles. Osterbrock and Shuder (1982) attribute the excess emission to Fe II multiplet 42 emission at $\lambda \lambda 4924,5018$ arising in the broad-line clouds. Both Oke and Lauer (1979) and Phillips (1978) show that the shape of the Fe II blends at $\lambda 4570$ and $\lambda \lambda 5190,5320$ are consistent with the Fe II emission having the same profiles as $\mathrm{H} \beta$. Thus, the breadth of the 


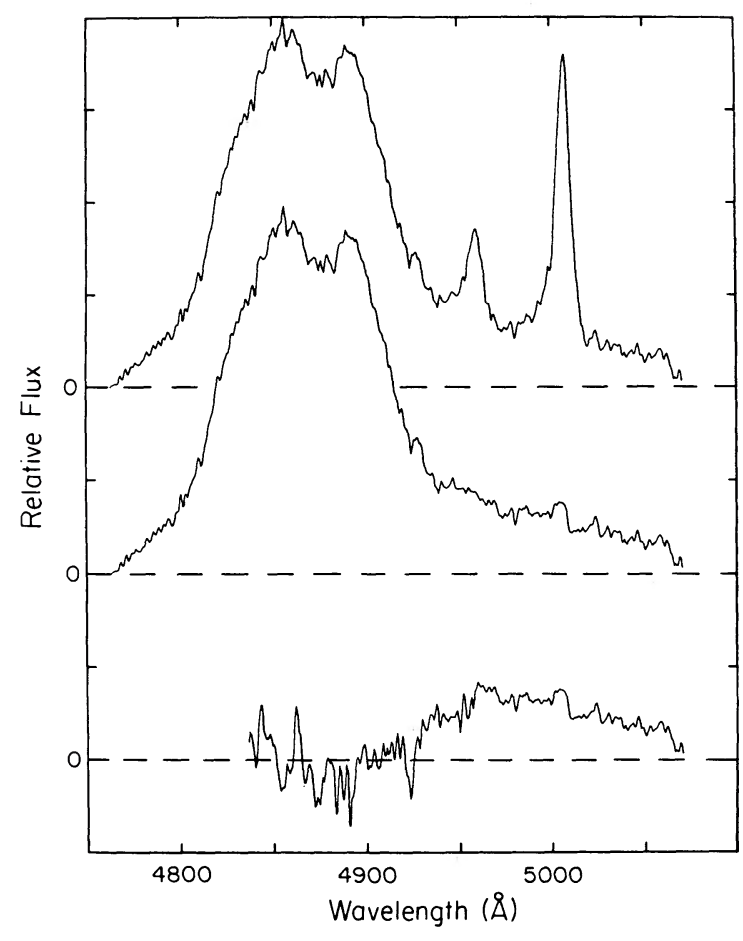

FIG. 2. A summary of the procedure used to isolate the extended red wing of $\mathrm{H} \beta$. Top: Observed $\mathrm{H} \beta$ profile; middle: profile corrected for [O III] $\lambda \lambda$ 4959, 5007; bottom; result of subtracting scaled $\mathrm{H} \gamma$ profile from the middle profile.

wing can ostensibly be explained. It has also been suggested (e.g., Shields 1978; Mathews 1982) that for models of the broad-line region in which all of the emitting clouds do not have the same electron density, some of the clouds may be of sufficiently low density such that [O III] emission is not effectively quenched. In the case of Mathews radiatively accelerated "pancake" clouds, broad, flat-topped emission with widths roughly equal to that of the broad lines are expected.

In order to investigate the nature of the red wing, it was isolated from the surrounding emission lines in the following manner: the [O III] $\lambda \lambda 5959,5007$ profiles were isolated and subtracted by interpolating the $\mathrm{H} \beta$ profile under them. This process is fairly unambiguous at the $\mathrm{S} / \mathrm{N}$ of these data. It was then assumed that the $\lambda 5007$ profile is characteristic of the forbidden line emission and it was used to subtract the narrow component of the $\mathrm{H} \beta$ emission from the $\mathrm{H} \beta$ profile and the narrow component of $\mathrm{H} \gamma$ and [O III] $\lambda 4363$ emission from the broad $\mathrm{H} \gamma$ profile. The flux ratios adopted in this subtraction are $F(\mathrm{H} \beta$ narrow): $F(\lambda$ 5007)::1:12, $F(\mathrm{H} \gamma$ narrow): $F(\lambda 4363): F(\lambda \cdot 5007):: 1: 1: 20$. This results in $\mathrm{H} \beta$ and $\mathrm{H} \gamma$ profiles, which are approximately corrected for narrow-line emission. Two independent methods were used in the subtraction of the broad $\mathrm{H} \beta$ profile. First, the $\mathrm{H} \gamma$ profile was shifted into the velocity space of $\mathrm{H} \beta$ and used as a template broad-line profile. Due to its proxim- ity to the end of the spectrum, the continuum level in and beyond the blue wing of $\mathrm{H} \gamma$ is uncertain and therfore, the blue wings of the two profiles could not be compared satisfactorily. Therefore, $\mathrm{H} \gamma$ was scaled by a constant until the central portions and near-red wings of the two profiles agreed reasonably well. The scaled and shifted $\mathrm{H} \gamma$ profile was then subtracted from $\mathrm{H} \beta$, isolating the spectrum of the red wing of $\mathrm{H} \beta$. The results of each stage of this process are displayed in Fig. 2.

It must be noted that this procedure suffers from several possible sources of error. Clearly the placement of the continuum affects the wings of the profile much more on a percentage basis than they do the parts of the profile near line center. The continuum level is particularly difficult to establish in the case of $\mathbf{H} \gamma$ due to its proximity to the end of the scan. Although the flux calibration should be accurate over the entire spectral range, the limited number of pixels observed shortward of $\mathrm{H} \gamma$ make the placement of the continuum extremely difficult. Furthermore, emissions from multiplets 37 and 38 of $\mathrm{Fe}$ II between $\mathrm{H} \gamma$ and $\mathrm{H} \beta$ also make the location of the continuum longward of $\mathrm{H} \gamma$ uncertain. Finally, the $\mathrm{H} \gamma$ profile itself is contaminated by an unknown amount of Fe II multiplet 27 emission. None of these problems is easily addressed.

As a consequence of the difficulties discussed above, a second attempt to isolate the red wing of $\mathrm{H} \beta$ was made. In this case it was assumed that the wings of the broad $\mathrm{H} \beta$ emission are symmetric. The blue wing of the $\mathrm{H} \beta$ profile was reflected about a point such that this reflected wing was in agreement with the "near-red" wing (i.e., between $\sim 4890$ and $4915 \AA$ rest). The position of the print of reflection was $\sim 380 \mathrm{~km} / \mathrm{s}$ longward of line center in the rest frame of the [O III] lines. The resultant profile is remarkably free of the problems discussed above. A similar procedure has been used in the study of the line profiles of QSOs and active galaxies by Wilkes (1983) and Meyers and Peterson (1983). The synthetic profile was then subtracted from the observed $\mathrm{H} \beta$ profile. The results of this subtraction are discussed below.

In order to investigate the veracity of the proposed sources of the emission, synthetic blends were generated and compared with the observed feature following the method discussed by Wilkes and Carswell (1982). In the first case, the $\mathrm{H} \gamma$ profile, and in the second case, the symmetric $\mathrm{H} \beta$, was used as a template for both $\mathrm{Fe}$ II and broad [O III] emission. Clearly this is not appropriate for the [O III] emission but this avoids the problem of selecting a theoretical shape for the lines. Both profiles are roughly rectangular in shape and so the comparison made below using it as a template profile is probably a reasonable approximation to the radiative acceleration model of Mathews (1982) in which any broad [O III] emission would be roughly rectangular. The template profile was shifted, in turn, into the velocity space of the Fe II and [O III] lines and scaled by their expected strengths in the optically thick case $(\mathrm{Fe}$ II $\lambda$ 5018: $\lambda$ 4024::1:1, [O III] $\lambda$ 5007: $\lambda$ 4959::3:1). The re- 


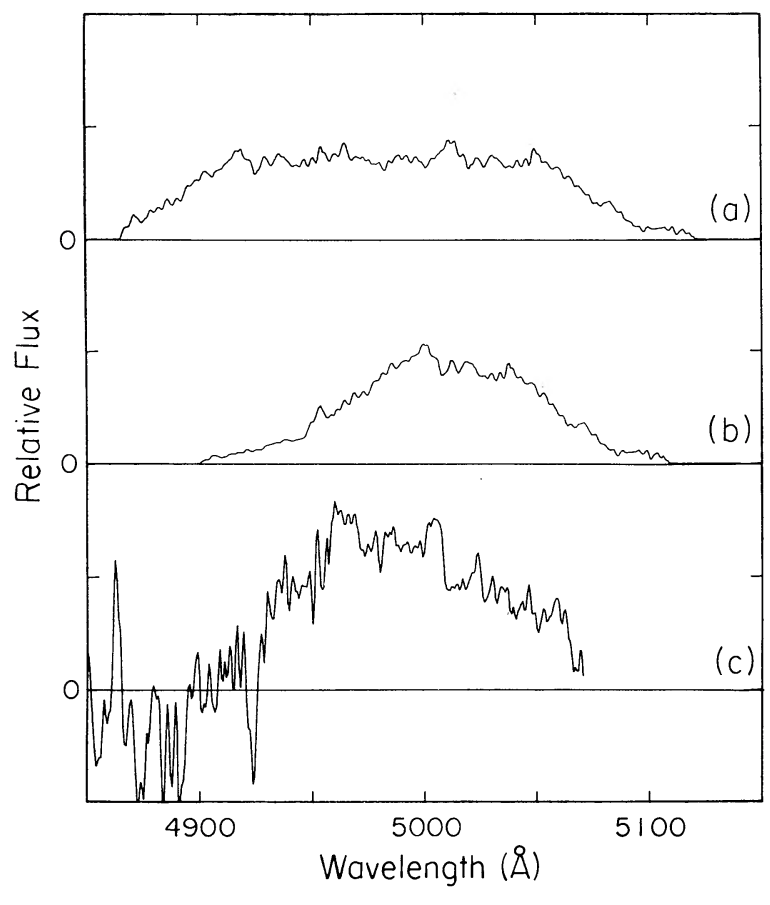

FIG. 3. Comparison of the excess emission in the extended red wing of $\mathrm{H} \beta$ (panel c) with synthetic features simulation (a) Fe II multiplet 42 emission, and (b) broad [O III] $\lambda \lambda 4959,5007$ emission. In this figure a scaled $\mathrm{H} \gamma$ profile was used to remove the broad $\mathrm{H} \beta$ emission and as a template for the synthesis.

sults are presented in Figs. 3 and 4 for the first and second cases, respectively. Panels (a) depict the synthetic Fe II blend; Panels (b) show the [O III] blend; and Panels (c) show the observed excess emission. The strengths of the synthetic blends were adjusted so that they were in reasonable agreement with the observed features at a wavelength of $\sim 5200 \AA$.

Due to the uncertainty in the choice of a template profile, it is not possible to make any firm conclusions but the following observations are presented for consideration: Fe II $\lambda \lambda 4924,5018$ are certainly present in emission, based on the strength of the other Fe II emission and the direct identification of $\lambda 4924$ and $\lambda 5018$ [see Panel (c) of Fig. 4]. However, the synthetic blend is a poor match to the observed feature; the synthetic feature is much broader than the observed one, which extends $\sim 50 \AA$ further blueward. The [O III] blends match more satisfactorily, particularly when using the symmetric $\mathrm{H} \beta$ template. In the case where the $\mathrm{H} \gamma$ template was used, the [O III] synthetic blend is not strong enough at shorter wavelengths. Although part of the discrepancies are certainly due to intrinsic differences between the real and template profiles, it appears likely that the observed feature is a combination of both $\mathrm{Fe}$ II and [O III] emission.

\section{SUMMARY AND CONCLUSIONS}

(1) A high-quality $\mathrm{H} \beta$ line profile for Akn 120 is presented for use as a benchmark for future line profile studies.

(2) The extended red wing of $\mathrm{H} \beta$ is probably not due solely to Fe II multiplet 42 emission where the individual transitions are emitted with the same profile as the Balmer lines. Some broad [O III] emission may be present.

(3) There are apparently two features in the $\mathrm{H} \beta$ profile at -1300 and $+1800 \mathrm{~km} / \mathrm{s}$ with respect to line center in the rest frame of the forbidden lines. The redward feature, and probably the blueward feature as well, have been stable in velocity for more than six years, though their relative strengths have varied.

It is interesting to speculate on the origin of the latter features. As noted by Peterson et al. (1983), they are probably not due to irregularities of excitation of the broad-line region caused by temporal variation of the continuum source as has been suggested by Capriotti, Foltz, and Peterson (1982). Possibly they indicate the presence of a kinematic component to the BLR distinct from that giving rise to the roughly logarithmic core and wings of the profile-e.g., a rotating disk or counterposed ejection of material. From symmetry consideration it is difficult to understand how the apparent $V / R$

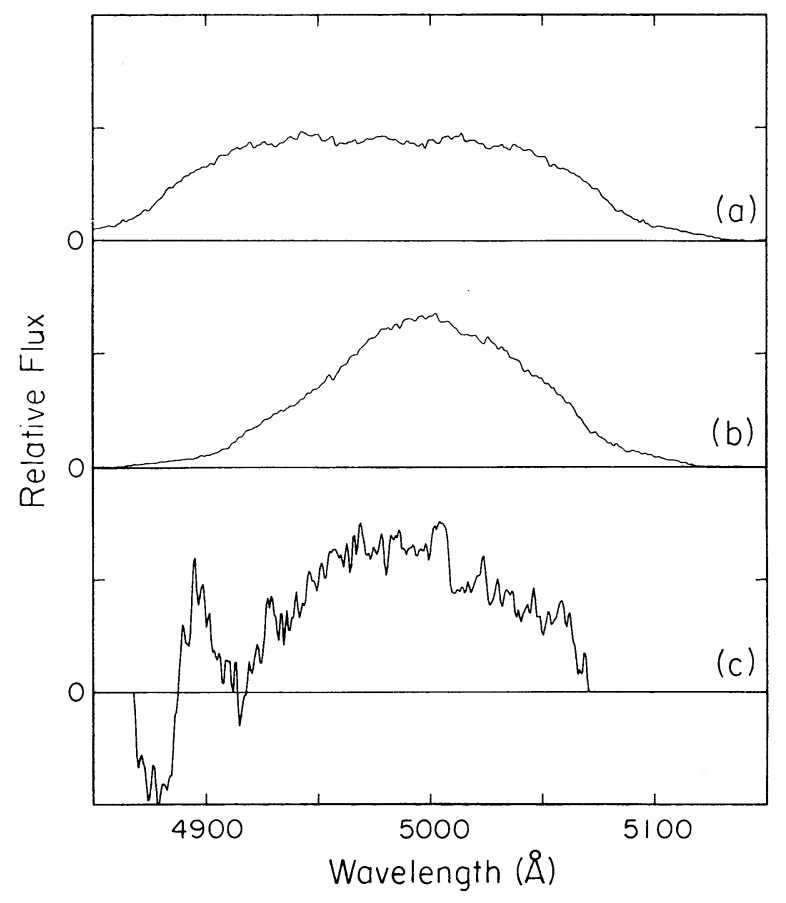

Fig. 4. Comparison of the extended red wing of $\mathrm{H} \beta$ as in Fig. 3. In this case the template was a synthetic $\mathrm{H} \beta$ profile generated as described in the text. 
asymmetry could be produced by a rotating structure alone. Since Akn 120 appears nearly face-on on the POSS, it is tempting to speculate that the material giving rise to this emission is being ejected parallel to the rotation axis of the galaxy. Perhaps some information as to whether such an additional component exists could be obtained by comparison of $\mathrm{H} \beta$ with $\mathrm{C}$ IV $\lambda 1550$ and $\operatorname{Mg}$ II $\lambda 2798$ profiles obtained with the space telescope or possibly IUE.

This research was supported by the National Science Foundation under Grants AST81-09025 to Steward Observatory and AST81-17095 to the Ohio State University. B. J. W. gratefully acknowledges the support of NATO through the SERC. We are grateful for the comments of an anonymous referee.

\section{REFERENCES}

Blandford, R. D., and McKee, C. F. (1982). Astrophys. J. 255, 419. Capriotti, E. R., Foltz, C. B., and Peterson, B. M. (1982). Astrophys. J. 261, 35.

Foltz, C. B., Peterson, B. M., Capriotti, E. R., Byard, P. L., Bertram, R., and Lawrie, D. G. (1982). Astrophys. J. 250, 508.

Mathews, W. G. (1982). Astrophys. J. 252, 39.

Meyers, K. A., and Peterson, B. M. (1983). In preparation.

Oke, J. B. (1974). Astrophys. J. Suppl. 27, 21.

Osterbrock, D. E., and Phillips, M. M. (1977). Publ. Astron. Soc. Pac. 89, 251.
Osterbrock, D. E., and Shuder, J. M. (1982). Astrophys. J. Suppl. 49, 149.

Peterson, B. M., Foltz, C. B., Miller, H. R., Wagner, R. M., Crenshaw, D. M., Meyers, K. A., and Byard, P. L. (1983). Astron. J. 88, 926.

Phillips, M. M. (1978). Astrophys. J. Suppl. 38, 187.

Wilkes, B. J., and Carswell, R. F. (1982). Mon. Not. R. Astron. Soc. 201, 645.

Wilkes, B. J. (1983). Submitted for publication. 